Case Report

\title{
Papillary thyroid carcinoma of thyroglossal duct cyst; a case report and review of the literature
}

\author{
Shelke VN ${ }^{1}$, Raut $\mathrm{WK}^{1}$ \\ ${ }^{1}$ Department of Pathology, Shri. Vasantrao Naik Government Medical College, Yavatmal, Maharashtra, India.
}

\author{
Keywords: \\ Papillary thyroid \\ carcinoma; \\ Thyroglossal duct Cyst
}

\begin{abstract}
Thyroglossal duct remnants are the most common midline neck swellings, but malignancy arising in them is a rare finding. Among the carcinomas arising in the thyroglossal duct cyst, papillary thyroid carcinoma is the commonest. Most of the cases of papillary thyroid carcinoma of thyroglossal duct cyst are asymptomatic and clinically diagnosed as thyroglossal duct cyst. It may remain undetected on fine needle aspiration cytology and ultrasonography; therefore diagnosis is established only after pathologic evaluation of biopsy. Hence careful histopathological examination of the excised specimen with high level of suspicion is mandatory in these cases. We are report a case of papillary thyroid carcinoma of thyroglossal duct cyst in a 30 -year-old female.
\end{abstract}

\section{INTRODUCTION}

Although thyroglossal duct cyst (TDC) is the most common developmental anomaly of the thyroid gland, carcinomas are found in approximately $1 \%$ of these patients. ${ }^{1}$ The first description of a neoplasm in a TDC was given by Brentano in 1911 and Uchermann in $1915 .^{2}$ Papillary thyroid carcinoma (PTC) comprises 75 to $85 \%$ of the tumors reported. The cysts are usually asymptomatic and the presentation of the patient with carcinoma is indistinguishable from the common cyst. Thus carcinoma is not suspected preoperatively and most cases are clinically diagnosed as TDC. Sometimes diagnosis is even not possible on fine needle aspiration cytology (FNAC) and ultrasonography (USG). ${ }^{1-4}$ We report

\author{
Correspondence: \\ Dr. Vijay N. Shelke, $M D$ \\ Department of Pathology, Shri. Vasantrao Naik Government Medical \\ College, Yavatmal, Maharashtra, India. \\ Email: vijaynshelke@yahoo.com
}

a case of papillary thyroid carcinoma of TDC in a 30-yearold female.

\section{CASE REPORT}

\section{Clinical Summary}

A 30-year-old female presented with the complaint of an anterior midline neck swelling since 10 years without any symptoms. The physical examination revealed a midline smooth, painless, cystic swelling of size $8 \times 4 \times 3 \mathrm{~cm}$ at the level of hyoid bone. Swelling moved on swallowing and on tongue protrusion. The thyroid gland was normal and no lymph nodes were found on palpation. Thyroid function tests were within normal limits. USG and FNAC of the swelling were suggestive of TDC. With the clinical diagnosis of TDC, patient underwent simple excision of the cyst. No thyroid abnormality was noted during clinical examination. 


\section{Pathologic Findings}

\section{Cytological findings}

The aspirate was fluid, which on microscopic examination revealed few macrophages and lymphocytes on the background of eosinophilic material. Neither epithelial cells nor malignant cells were seen. Taking clinical findings into consideration, the FNAC diagnosis was given as TDC.

\section{Histopathological findings}

Gross Appearance: The resected specimen was a single cystic mass covered by skin, together measured $10 \times 4 \times 3$ $\mathrm{cm}$. Inner aspect of the cyst wall was smooth and white. In an area, cyst wall was thickened and irregular forming an indistinct mass. The cut surface of the mass was light brown to pale in color. Cyst wall also showed a small colloid nodule (fig.1A). Specimen was examined thoroughly and multiple sections were taken for histopathological study.

Microscopic findings: Sections from the cyst showed the fibrocollagenous cyst wall lined by low columnar to cuboidal epithelium. Sections from the mass showed a tumor composed of round to cuboidal cells arranged in papillae, follicles and clusters. Cellular crowding with nuclear overlapping, clearing, and grooving were noted (fig.1B). Occasional nuclear pseudoinclusion was noted. Foci of calcification and occasional psammoma body were seen.

On the basis of gross and microscopic findings the histopathological diagnosis was 'Papillary thyroid carcinoma $\square$ of TDC.

\section{DISCUSSION}

Carcinoma arising in TDC is a rare finding and found in approximately $1 \%$ of these cysts. About 250 cases are reported in the literature. ${ }^{5}$ Etiology remains unclear but de novo origin is generally proposed, although metastatic theory has been also suggested. ${ }^{1,6}$ PTC arising in TDC is seen most frequently in young woman with a sex ratio of 1.5:1. Gross and microscopic features are same as its counterpart in the thyroid gland. ${ }^{1}$

Though FNAC has been proved to be useful diagnostic technique for establishing a preoperative diagnosis, but most of the lesions of PTC in TDC are very small or may be microscopic so that it can be missed on FNAC as well as on USG. Thus in most cases the diagnosis is established only after excision of a clinically benign TDC; and thus these carcinomas become an incidental finding. Therefore thorough and meticulous sampling with careful gross and microscopic examination is mandatory. For preoperative diagnosis, proper clinical examination and investigations like USG, CT-Scan, and thyroid scan are recommended. ${ }^{5}$

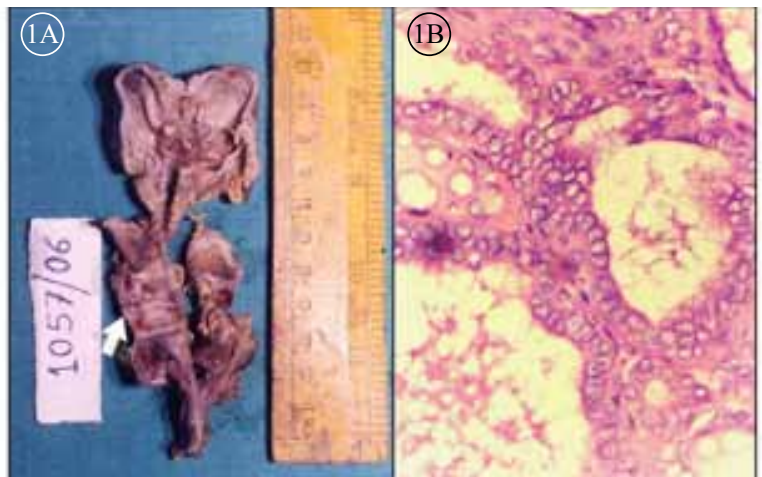

Figure 1: (A) Gross specimen showing thyroglossal duct cyst with solid tumor mass (arrow) and colloid nodule.

(B) Photomicrograph showing nuclear features of papillary thyroid carcinoma-nuclear overlapping, clearing, and occasional grooving (HE stain, X400).

However controversies exist in relation to a rational and effective therapeutic approach. Some authors consider that the optimal surgical procedure for thyroglossal duct carcinoma is same as that for the benign cyst i.e. Sistrunck procedure. The main question is what to do with the thyroid gland. There is still controversy about thyroid removal for PTC of TDC, but all the patients should receive suppressive doses of thyroid hormone. ${ }^{4}$ Some authors recommend total thyroidectomy with removal of the tumor of the thyroglossal duct and the body of the hyoid bone, because the carcinoma may be multifocal and there may be lymphatic invasion of the thyroid ${ }^{7}$ Hence, the Sistrunk operation alone is sufficient for squamous carcinoma, but total thyroidectomy is recommended for differentiated thyroid carcinoma. ${ }^{8}$ The patient presenting with metastatic carcinoma should, of course, be treated with appropriate local resection, hormonal manipulation, thyroid ablation, and lymphadectomy as indicated by his/her age and sex, the cell type of tumor, and the extent of local and metastatic disease.

Papillary thyroid carcinoma in TDC has more indolent course than its thyroid homologue, and could therefore be singled out as a clinicopathologic entity. ${ }^{8}$ Prognosis is excellent as the cure rate is $95 \%{ }^{4}$ and mortality is only $2 \%$ at 5 years.

\section{CONCLUSION}

As prognosis is excellent in papillary thyroid carcinoma of TDC, the correct diagnosis is important for effective therapeutic approach and follow-up. Most of these patients are clinically asymptomatic and remain undetected on USG and FNAC. Diagnosis could be possible only after histopathological examination of the ressected specimen. Thus along with high index of suspicion, careful pathological examination is indicated in these cases. 


\section{REFERENCES}

1. Balalaa N, Megahed M, Ashari MA, Branicki F. Thyroglossal duct cyst papillary carcinoma. Case Rep Oncol 2011;29:39-43.

2. Kristensen S, Juul A, Moesner J. Thyroglossal cyst carcinoma. J Laryngol Otol 1984;98:1277-80.

3. Srinivasan R, Ranjini K, Vadhiraja BM. Primary papillary carcinoma of the thyroglossal duct cyst- a case report. Indian J Pathol Microbiol 2005;48:228-30.

4. Dedivitis RA, Guimaraes AV. Papillary thyroid carcinoma in thyroglossal duct cyst. Int Surg 2000;85:198-201.
5. Cannizzaro MA, Castanzo M, Fiorenza $\mathrm{G}$ et al. Papillary carcinoma in an isthmic thyroglossal duct cyst: clinical considerations. Chir Ital 2006;58:105-11.

6. Vera-Sempere F, Tuz J, Jaen J, Perolada JM et al. Papillary thyroid carcinoma arising in the wall of a thyroglossal duct cyst. Acta otorhinolaryngol Belg 1998;52:49-54.

7. Falvo L, Giacomelli L, Vanni B et al. Papillary thyroid corcinoma in thyroglossal duct cyst: case report and literature review. Int Surg 2006;91:141-6.

8. Falconieri G, Della libera D, Zanella M. Papillary thyroid carcinoma of thyroglossal duct cyst; comparative cytohistologic and immunochemical study of 2 new cases and review of literature. Int J Surg Pathol 2001;9:65-71. 